PROCEEDINGS OF THE

AMERICAN MATHEMATICAL SOCIETY

Volume 130, Number 4, Pages 939-949

S 0002-9939(01)06092-0

Article electronically published on November 9, 2001

\title{
INVARIANT IDEALS OF ABELIAN GROUP ALGEBRAS UNDER THE MULTIPLICATIVE ACTION OF A FIELD. I
}

\author{
D. S. PASSMAN AND A. E. ZALESSKIĬ \\ (Communicated by Lance W. Small)
}

\begin{abstract}
Let $D$ be a division ring and let $V=D^{n}$ be a finite-dimensional $D$-vector space, viewed multiplicatively. If $G=D^{\bullet}$ is the multiplicative group of $D$, then $G$ acts on $V$ and hence on any group algebra $K[V]$. Our goal is to completely describe the semiprime $G$-stable ideals of $K[V]$. As it turns out, this result follows fairly easily from the corresponding results for the field of rational numbers (due to Brookes and Evans) and for infinite locally-finite fields. Part I of this work is concerned with the latter situation, while Part II deals with arbitrary division rings.
\end{abstract}

\section{INTRODUCTION I}

In a long series of papers (see $[\mathbf{Z}]$ ), the second author studied the ideal structure of various complex group algebras $\mathbb{C}[\mathfrak{H}]$, with $\mathfrak{H}$ an infinite locally-finite simple group. It now appears that the next family of groups to be considered will have the form $\mathfrak{H}=V \rtimes \mathfrak{G}$, where $V$ is an elementary abelian group and $\mathfrak{G}$ is an infinite locally-finite "almost" simple group (see [PZ1). For example, $\mathfrak{G}$ might be the group $\mathrm{GL}_{n}(F)$ where $F$ is an infinite locally-finite field, and $V$ could be a suitable finitedimensional $F$-vector space viewed multiplicatively. Note that a field is locally finite or absolute if every finite subset generates a finite subfield. In other words, $F$ is locally finite precisely when it is a subfield of the algebraic closure of a finite field. Now $\mathfrak{G}$ acts on $V$, so it acts on $\mathbb{C}[V]$. Thus, a necessary first ingredient in the ideal structure of $\mathbb{C}[\mathfrak{H}]$ is a description of the $\mathfrak{G}$-stable ideals of $\mathbb{C}[V]$. Indeed, since $\mathfrak{G}$ might contain an isomorphic copy of $F^{\bullet}$, the multiplicative group of $F$ acting naturally on the $F$-vector space $V$, it is appropriate to consider the $F^{\bullet}$-stable ideals of $\mathbb{C}[V]$. For this problem, there is really no need to restrict our attention to the complex field. Thus, we let $K$ be any field, although we usually require that its characteristic be different from that of $F$.

Let $V$ be a multiplicative abelian group and let $K[V]$ denote its group algebra over the field $K$. If $A$ is a subgroup of $V$, then there exists a natural epimorphism $K[V] \rightarrow K[V / A]$ and we let $\omega(A ; V)=\omega_{K}(A ; V)$, the augmentation ideal of $A$ in $V$, denote its kernel. Thus, $\omega(A ; V)$ is the $K$-linear span of all elements of the form $(1-a) v$ with $a \in A$ and $v \in V$. If $G$ is a group which acts as automorphisms on

Received by the editors October 3, 2000.

2000 Mathematics Subject Classification. Primary 16S34, 12E20.

The first author's research was supported in part by NSF Grant DMS-9820271. Much of this work was performed during the second author's visit to the University of Wisconsin-Madison, made possible by the financial support of EPSRC. 
$V$, then $G$ also acts on $K[V]$, and it is clear that $A$ is a $G$-stable subgroup of $V$ if and only if $\omega(A ; V)$ is a $G$-stable ideal of $K[V]$. The main result of this paper is

Theorem A. Let $F$ be an infinite locally-finite field and let $V=F^{n}$ be a finitedimensional $F$-vector space, viewed multiplicatively. If $G=F^{\bullet}$, then $G$ acts on $V$ and hence on the group algebra $K[V]$. Suppose, in addition, that $\operatorname{char} K \neq \operatorname{char} F$. Then every $G$-stable ideal of $K[V]$ can be written uniquely as a finite irredundant intersection $\bigcap_{i=1}^{k} \omega\left(A_{i} ; V\right)$ of augmentation ideals, where each $A_{i}$ is an $F$-subspace of $V$. As a consequence, the set of these $G$-stable ideals is Noetherian.

Note that, if $V$ is a torsion abelian group having no elements of order equal to the characteristic of $K$, then $K[V]$ is a commutative von Neumann regular algebra (see [P. Theorem 1.1.5]). It follows that if $I, J \triangleleft K[V]$, then $I \cap J=I J$. In particular, finite products and finite intersections of ideals coincide here. Furthermore, every ideal of $K[V]$ is semiprime. In view of this latter comment, it is clear that Theorem A is the locally-finite analog (with some enhancements) of [BE, Proposition 6] which studies finite-dimensional rational vector spaces.

\section{Generalities}

Let $G$ be a group of operators on the abelian group $V$. We start with a simple observation.

Lemma 1.1. Let $W$ be a subgroup of $V$. Then:

(i) $K[W] \cap \omega(A ; V)=\omega(W \cap A ; W)$

(ii) If $I=\bigcap_{i \in \mathcal{I}} \omega\left(A_{i} ; V\right)$, then $K[W] \cap I=\bigcap_{i \in \mathcal{I}} \omega\left(W \cap A_{i} ; W\right)$.

Proof. Part (i) follows since the restriction to $K[W]$ of the map $K[V] \rightarrow K[V / A]$ is clearly $K[W] \rightarrow K[W /(W \cap A)]$. Part (ii) is immediate from (i).

Now we say that $\left\{\left(V_{i}, G_{i}\right) \mid i=1,2, \ldots\right\}$ is a local system for $(V, G)$ if:

(1) $V_{1} \subseteq V_{2} \subseteq \cdots \subseteq V_{n} \subseteq \cdots$ and $\bigcup_{1}^{\infty} V_{i}=V$.

(2) $G_{1} \subseteq G_{2} \subseteq \cdots \subseteq G_{n} \subseteq \cdots$ and $\bigcup_{1}^{\infty} G_{i}=G$.

(3) $G_{i}$ stabilizes $V_{i}$ and both of these subgroups are finite.

The next lemma shows that the property of being an intersection of augmentation ideals can be lifted from a local system to the entire group.

Lemma 1.2. Let $\left\{\left(V_{i}, G_{i}\right) \mid i=1,2, \ldots\right\}$ be a local system for $(V, G)$, and let $I$ be a $G$-stable ideal of $K[V]$. Suppose that, for each $i$, the ideal $K\left[V_{i}\right] \cap I$ of $K\left[V_{i}\right]$ is an intersection of augmentation ideals of $G_{i}$-stable subgroups of $V_{i}$. Then $I$ is an intersection of augmentation ideals of $G$-stable subgroups of $V$.

Proof. For each $i$, let $\mathbb{A}_{i}$ be the set of all families $\mathfrak{A}$ of $G_{i}$-stable subgroups of $V_{i}$ such that $I_{i}=K\left[V_{i}\right] \cap I=\bigcap_{A \in \mathfrak{A}} \omega\left(A ; V_{i}\right)$. By assumption, each $\mathbb{A}_{i}$ is nonempty. Furthermore, since $V_{i}$ is a finite group, it is clear that each $\mathfrak{A}$, as above, is finite, and so also is each $\mathbb{A}_{i}$. Since

$$
I_{i}=K\left[V_{i}\right] \cap I=K\left[V_{i}\right] \cap\left(K\left[V_{i+1}\right] \cap I\right)=K\left[V_{i}\right] \cap I_{i+1},
$$

Lemma 1.1(ii) implies that there is an intersection map $\operatorname{Int}_{i}: \mathbb{A}_{i+1} \rightarrow \mathbb{A}_{i}$ given by

$$
\left\{A_{1}, A_{2}, \ldots, A_{t}\right\} \mapsto\left\{V_{i} \cap A_{1}, V_{i} \cap A_{2}, \ldots, V_{i} \cap A_{t}\right\} .
$$

Thus $\mathbb{T}=\left\{\mathbb{A}_{i}, \operatorname{Int}_{i} \mid i=1,2, \ldots\right\}$ can be viewed as a tree with $\mathbb{A}_{i}$ being the set of elements at level $i$ and with $\operatorname{Int}_{i}$ describing the branches from level $i+1$ to level $i$. 
Since each $\mathbb{A}_{i}$ is finite and nonempty, there exists an infinite branch in this tree. In other words, we can choose fixed $\mathfrak{A}_{i} \in \mathbb{A}_{i}$ such that $\operatorname{Int}_{i}: \mathfrak{A}_{i+1} \mapsto \mathfrak{A}_{i}$ for all $i$. Say $\mathfrak{A}_{i}=\left\{A_{i, 1}, A_{i, 2}, \ldots, A_{i, t_{i}}\right\}$.

Since $\operatorname{Int}_{i}: \mathfrak{A}_{i+1} \mapsto \mathfrak{A}_{i}$, there is a second intersection map int ${ }_{i}: \mathfrak{A}_{i+1} \rightarrow \mathfrak{A}_{i}$ given by int ${ }_{i}: A_{i+1, j} \mapsto V_{i} \cap A_{i+1, j}$. Thus $\mathcal{T}=\left\{\mathfrak{A}_{i}\right.$, int $\left._{i} \mid i=1,2, \ldots\right\}$ can be viewed as a tree with $\mathfrak{A}_{i}$ being the set of elements at level $i$ and with int ${ }_{i}$ describing the branches from level $i+1$ to level $i$. Again, each $\mathfrak{A}_{i}$ is finite and nonempty. Furthermore, since $\operatorname{Int}_{i}\left(\mathfrak{A}_{i+1}\right)=\mathfrak{A}_{i}$, this tree has the additional property that each node at level $i$ is the image of a node at level $i+1$. Equivalently, each $A_{i, j}$ is equal to $V_{i} \cap A_{i+1, j^{\prime}}$ for some subscript $j^{\prime}$.

Now let $\mathfrak{B}$ be any full branch of the tree $\mathcal{T}$. Then $\mathfrak{B}$ has nodes

$$
A_{1, j_{1}} \subseteq A_{2, j_{2}} \subseteq \cdots \subseteq A_{n, j_{n}} \subseteq \cdots
$$

for suitable subscripts $j_{1}, j_{2}, \ldots, j_{n}, \ldots$, and we let $B_{\mathfrak{B}}=\bigcup_{i} A_{i, j_{i}}$. We claim that each $B_{\mathfrak{B}}$ is a $G$-stable subgroup of $V$ and that $I$ is equal to the ideal $J=$ $\bigcap_{\mathfrak{B}} \omega\left(B_{\mathfrak{B}} ; V\right)$, where the intersection is over all such branches $\mathfrak{B}$. This will certainly yield the result.

To start with, it is clear that each $B_{\mathfrak{B}}$ is a subgroup of $V$. Furthermore, for any $k \geq i$, we know that $G_{k} \supseteq G_{i}$ and that $A_{k, j_{k}}$ is $G_{i}$-stable. Thus $B_{\mathfrak{B}}$ is $G_{i}$-stable for all $i$, and hence it is $G$-stable. Next, by definition of the map $\operatorname{int}_{i}$, if $B_{\mathfrak{B}}$ is defined as above, then $V_{i} \cap A_{k, j_{k}}=A_{i, j_{i}}$ for all $k \geq i$ and hence $V_{i} \cap B_{\mathfrak{B}}=A_{i, j_{i}}$. Thus, by Lemma 1.1(ii) and the fact that each $A_{i, j}$ is a member of some full branch $\mathfrak{B}$, we have

$$
J_{i}=K\left[V_{i}\right] \cap J=\bigcap_{\mathfrak{B}} \omega\left(V_{i} \cap B_{\mathfrak{B}} ; V_{i}\right)=\bigcap_{j=1}^{t_{i}} \omega\left(A_{i, j} ; V_{i}\right)=K\left[V_{i}\right] \cap I=I_{i},
$$

where the latter uses the fact that $\left\{A_{i, 1}, A_{i, 2}, \ldots, A_{i, t_{i}}\right\}=\mathfrak{A}_{i} \in \mathbb{A}_{i}$. Since $V$ is the ascending union of the subgroups $V_{i}$, it now follows from $J_{i}=I_{i}$ that $J=I$, and the lemma is proved.

As is readily apparent, the preceding argument only shows that $I$ is an infinite intersection of augmentation ideals. Even so, this turns out to be a fairly powerful conclusion. For example, if $I$ is properly smaller than $\omega(V ; V)$, then it follows from the above that $I \subseteq \omega(A ; V)$ for some proper $G$-stable subgroup $A$ of $V$. To proceed further, it is necessary to recall some standard notation.

First, a $G$-stable ideal $I$ of $K[V]$ is said to be $G$-prime if the inclusion $J_{1} J_{2} \subseteq I$ implies that one of the $G$-stable ideals $J_{1}$ or $J_{2}$ is contained in $I$. Second, $K[V]$ is a $G$-prime algebra if $I=0$ is a $G$-prime ideal. In other words, $K[V]$ is $G$-prime if and only if the product of any two nonzero $G$-stable ideals is again nonzero. Finally, $B / A$ is a $G$-section of $V$ if $A \subset B$ are any two distinct $G$-stable subgroups of $V$.

Lemma 1.3. Suppose that all $G$-sections of $V$ are infinite. Then:

(i) $K[V]$ is a $G$-prime algebra.

(ii) If $A$ is a $G$-stable subgroup of $V$, then $\omega(A ; V)$ is a $G$-prime ideal of $K[V]$.

Proof. (i) Form the group $H=V \rtimes G$, and suppose that $I$ and $J$ are $G$-stable ideals of $K[V]$ with $I J=0$. Then $I^{\prime}=I \cdot K[H]$ and $J^{\prime}=J \cdot K[H]$ are two-sided ideals of $K[H]$ with $I^{\prime} J^{\prime}=0$. In particular, if $\Delta(H)$ is the f.c. center of $H$ and if $\theta: K[H] \rightarrow K[\Delta(H)]$ denotes the natural projection, then [P Theorem 4.2.9] implies that $\theta\left(I^{\prime}\right) \theta\left(J^{\prime}\right)=0$ and hence that $\theta(I) \theta(J)=0$. But all $G$-sections of $V$ 
are infinite, so it follows from $[\mathrm{P}$, Lemma 4.1.8] that $V \cap \Delta(H)$ must be torsion-free abelian. In particular, $K[V \cap \Delta(H)]$ is a domain, so $\theta(I) \theta(J)=0$ implies that either $\theta(I)=0$ or $\theta(J)=0$, and consequently that either $I=0$ or $J=0$.

(ii) Since $K[V] / \omega(A ; V)=K[V / A]$ and $V / A$ has the same $G$-structure as $V$, the result follows from part (i).

As a consequence, we can now easily describe the uniqueness aspects of finite intersections of augmentation ideals. If $I=\bigcap_{1}^{n} \omega\left(A_{i} ; V\right)$ and if $A_{1} \subseteq A_{2}$, then $\omega\left(A_{1} ; V\right) \subseteq \omega\left(A_{2} ; V\right)$ and $\omega\left(A_{2} ; V\right)$ is not needed. Thus, we say that the intersection is irredundant if $A_{i} \subseteq A_{j}$ implies that $i=j$.

Lemma 1.4. Suppose that all $G$-sections of $V$ are infinite. Let $A_{1}, A_{2}, \ldots, A_{n}$ and $B_{1}, B_{2}, \ldots, B_{m}$ be $G$-stable subgroups of $V$ and assume that

$$
I=\bigcap_{1}^{n} \omega\left(A_{i} ; V\right) \quad \text { and } \quad J=\bigcap_{1}^{m} \omega\left(B_{j} ; V\right)
$$

are irredundant intersections. Then $I \supseteq J$ if and only if each $A_{i}$ contains some $B_{j}$. In particular, if $I=J$, then $n=m$ and, by reordering the $B_{j}$ 's if necessary, we have $A_{i}=B_{i}$ for all $i$.

Proof. Suppose first that $I \supseteq J$. Then, for each subscript $i$, we have

$$
\omega\left(A_{i} ; V\right) \supseteq I \supseteq J \supseteq \prod_{1}^{m} \omega\left(B_{j} ; V\right) .
$$

Thus, since $\omega\left(A_{i} ; V\right)$ is a $G$-prime ideal of $K[V]$ by Lemma $1.3($ ii), we conclude that $\omega\left(A_{i} ; V\right) \supseteq \omega\left(B_{j} ; V\right)$ for some $j$. In particular, $A_{i} \supseteq B_{j}$. Conversely, suppose that each $A_{i}$ contains some $B_{j}$. Then, for each $i$, we have $\omega\left(A_{i} ; V\right) \supseteq \omega\left(B_{j} ; V\right) \supseteq J$ and hence $I=\bigcap_{i} \omega\left(A_{i} ; V\right) \supseteq J$.

Finally, suppose that $I=J$ and let $i$ be given. Then $I \supseteq J$, so $A_{i} \supseteq B_{j}$ for some $j$. On the other hand, $J \supseteq I$, so $B_{j} \supseteq A_{i^{\prime}}$ for some $i^{\prime}$. Thus $A_{i} \supseteq B_{j} \supseteq A_{i^{\prime}}$ and the irredundancy of the $I$-intersection implies that $i=i^{\prime}$ and $A_{i}=B_{j}$. We have therefore shown that

$$
\left\{A_{1}, A_{2}, \ldots, A_{n}\right\} \subseteq\left\{B_{1}, B_{2}, \ldots, B_{m}\right\} .
$$

By symmetry, we get the reverse inclusion, and the result follows.

We will also need the following variant of the above.

Lemma 1.5. Assume that all $G$-sections on $V$ are infinite. Let $I$ be a $G$-stable ideal of $K[V]$, and let $X \subseteq Y$ be $G$-stable subgroups of $V$. Suppose that $I \cap K[X]=$ $\bigcap_{i=1}^{n} \omega\left(A_{i} ; X\right)$ and $I \cap K[Y]=\bigcap_{j=1}^{m} \omega\left(B_{j} ; Y\right)$ are finite irredundant intersections, where the $A_{i}$ and $B_{j}$ are $G$-stable subgroups of $X$ and $Y$, respectively. Then each $B_{j}$ contains some $A_{j^{\prime}}$, and each $A_{i}$ can be written as $A_{i}=B_{i^{\prime}} \cap X$ for some $B_{i^{\prime}}$.

Proof. By assumption and Lemma 1.1(ii), we have

$$
\bigcap_{i=1}^{n} \omega\left(A_{i} ; X\right)=I \cap K[X]=(I \cap K[Y]) \cap K[X]=\bigcap_{j=1}^{m} \omega\left(B_{j} \cap X ; X\right) .
$$

Since $\omega\left(B_{j} \cap X ; X\right) \supseteq \bigcap_{i=1}^{n} \omega\left(A_{i} ; X\right)$, it follows from Lemma 1.3(ii) that $B_{j} \supseteq$ $B_{j} \cap X \supseteq A_{j^{\prime}}$ for some $j^{\prime}$. Conversely, if we eliminate unnecessary terms from $\bigcap_{j=1}^{m} \omega\left(B_{j} \cap X ; X\right)$, then we have a finite irredundant intersection, so Lemma 1.4 implies that $A_{i}=B_{i^{\prime}} \cap X$ for some $i^{\prime}$. 
Next, we introduce an assumption to guarantee that any arbitrary intersection of augmentation ideals can be reduced to a finite intersection.

Lemma 1.6. Assume that $V$ has a finite-length composition series as a $G$-module. Let $\mathfrak{A}$ be a nonempty family of $G$-stable subgroups of $V$ and let $I=\bigcap_{A \in \mathfrak{A}} \omega(A ; V)$. Then $I$ can be written as a finite intersection of augmentation ideals $\omega(B ; V)$ with each $B$ a $G$-stable subgroup of $V$.

Proof. For convenience we can assume that $V \in \mathfrak{A}$ since $\omega(V ; V) \supseteq \omega(A ; V)$ for all $A$. We proceed by induction on the $G$-composition length of $V$, the result being trivial if the length is 0 . Now if $I=0$, then $I=\omega(1 ; V)$ and we are done. So assume that $I \neq 0$ and choose $0 \neq \gamma=\sum_{0}^{n} k_{i} x_{i} \in I$ with $x_{0}=1$ and $0 \neq k_{0} \in K$. Let $X_{i}$ be the $G$-stable subgroup of $V$ generated by $x_{i}$ for $i=1,2, \ldots, n$, and let $\mathfrak{A}_{i}=\left\{A \in \mathfrak{A} \mid A \supseteq X_{i}\right\}$. Note that each $\mathfrak{A}_{i}$ is nonempty since $V \in \mathfrak{A}_{i}$. We claim that $\mathfrak{A}=\bigcup_{1}^{n} \mathfrak{A}_{i}$.

To this end, let $A \in \mathfrak{A}$. Then

$$
\gamma=\sum_{0}^{n} k_{i} x_{i} \in I \subseteq \omega(A ; V)
$$

so $x_{0}=1$ and $k_{0} \neq 0$ implies that $x_{i} \in A$ for some $i \neq 0$. But $A$ is $G$-stable, so $X_{i} \subseteq A$ and hence $A \in \mathfrak{A}_{i}$, as required. Now set $I_{i}=\bigcap_{A \in \mathfrak{A}_{i}} \omega(A ; V)$, so that $I=I_{1} \cap I_{2} \cap \cdots \cap I_{n}$. It clearly suffices to show that each $I_{i}$ is a finite intersection of suitable $\omega(B ; V)$.

For this, note that $A \supseteq X_{i}$ implies that $\omega(A ; V) \supseteq \omega\left(X_{i} ; V\right)$ and that

$$
\omega(A ; V) / \omega\left(X_{i} ; V\right)=\omega\left(A / X_{i} ; V / X_{i}\right) \subseteq K\left[V / X_{i}\right] .
$$

Thus

$$
I_{i} / \omega\left(X_{i} ; V\right)=\bigcap_{A \in \mathfrak{A}_{i}} \omega\left(A / X_{i} ; V / X_{i}\right)
$$

Now, $X_{i} \neq 1$, so $V / X_{i}$ has smaller $G$-composition length than $V$. Thus, by induction, $I_{i} / \omega\left(X_{i} ; V\right)$ can be written as a finite intersection $\bigcap_{j} \omega\left(B_{i, j} / X_{i} ; V / X_{i}\right)$ where each $B_{i, j}$ is a $G$-stable subgroup of $V$ containing $X_{i}$. Thus $I_{i}=\bigcap_{j} \omega\left(B_{i, j} ; V\right)$ and we have the finite intersection

$$
I=\bigcap_{i} I_{i}=\bigcap_{i, j} \omega\left(B_{i, j} ; V\right),
$$

as required.

A slight generalization of part of the preceding argument yields the following result which requires no assumption on the $G$-module structure of $V$.

Lemma 1.7. Let $\mathfrak{A}$ be a nonempty family of subgroups of $V$, let $I=\bigcap_{A \in \mathfrak{A}} \omega(A ; V)$, and let $\alpha \in I$. Then we can write $\mathfrak{A}$ as a finite union $\mathfrak{A}=\mathfrak{A}_{1} \cup \mathfrak{A}_{2} \cup \cdots \cup \mathfrak{A}_{m}$, depending upon $\alpha$, so that if $A_{i}=\bigcap_{A \in \mathfrak{A}_{i}} A$, then $\alpha \in \bigcap_{1}^{m} \omega\left(A_{i} ; V\right)$.

Proof. Let $S$ be the support of $\alpha$, namely the finite set of elements of $V$ which appear in the representation of $\alpha \in K[V]$. Note that each $A \in \mathfrak{A}$ partitions $V$ into disjoint $A$-cosets, and hence each such $A$ gives rise to a partition of $S$. But $S$ is finite, so there are only finitely many possible partitions. Thus, we can write $\mathfrak{A}=\mathfrak{A}_{1} \cup \mathfrak{A}_{2} \cup \cdots \cup \mathfrak{A}_{m}$ as a finite union of nonempty sets, where all $A$ 's in a fixed $\mathfrak{A}_{i}$ yield the same partition $\mathcal{P}_{i}$ of $S$. If $A_{i}=\bigcap_{A \in \mathfrak{A}_{i}} A$, it suffices to show that 
$\alpha \in \omega\left(A_{i} ; V\right)$. To this end, fix $i$ and write $\alpha=\alpha_{1}+\alpha_{2}+\cdots+\alpha_{k}$ where the $\alpha_{j}$ 's have support corresponding to the various subsets of $S$ in the partition $\mathcal{P}_{i}$. Now for any $A \in \mathfrak{A}_{i}$, we have $\alpha=\sum_{j} \alpha_{j} \in \omega(A ; V)$. Thus, since each $\alpha_{j}$ has support in precisely one coset of $A$ and since these cosets are disjoint, we conclude that $\alpha_{j} \in \omega(A ; V)$. In particular, the sum of the coefficients of each $\alpha_{j}$ is 0 , and since the support of $\alpha_{j}$ is contained in precisely one coset of $A_{i}=\bigcap_{A \in \mathfrak{A}_{i}} A$, we conclude that $\alpha_{j} \in \omega\left(A_{i} ; V\right)$. It follows that $\alpha \in \omega\left(A_{i} ; V\right)$, as required.

Next, we prove the expected Noetherian result.

Lemma 1.8. Assume that $V$ has a finite-length composition series as a $G$-module and that all composition factors are infinite. If $\mathcal{I}$ is the set of ideals of $K[V]$ which can be written as intersections of augmentation ideals of $G$-stable subgroups of $V$, then $\mathcal{I}$ satisfies the ascending chain condition.

Proof. It is clear from the assumption that every $G$-section of $V$ is infinite. Furthermore, Lemma 1.6 implies that every ideal in $\mathcal{I}$ can be written as a finite intersection of augmentation ideals of $G$-stable subgroups of $V$. Indeed, we can then assume that these intersections are irredundant and hence satisfy the uniqueness condition of Lemma 1.4. In other words, each $I \in \mathcal{I}$ corresponds uniquely to a finite irredundant set $\left\{A_{1}, A_{2}, \ldots\right\}$ of $G$-stable subgroups of $V$. Moreover, if $J$ corresponds to $\left\{B_{1}, B_{2}, \ldots\right\}$, then $I \supseteq J$ if and only if each $A_{i}$ contains some $B_{j}$.

Let $\mathbb{S}$ denote the collection of all finite irredundant sets $\left\{A_{1}, A_{2}, \ldots\right\}$ of $G$-stable subgroups of $V$, and define $\left\{B_{1}, B_{2}, \ldots\right\} \preceq\left\{A_{1}, A_{2}, \ldots\right\}$ if and only if each $A_{i}$ contains some $B_{j}$. In view of the comments of the preceding paragraph, it suffices to show that $\mathbb{S}, \preceq$ satisfies the ascending chain condition. To this end, for every $G$-stable subgroup $A$ of $V$, define the depth of $A, d(A)$, to equal the $G$-composition length of $V / A$, and note that this parameter is at most equal to the $G$-composition length of $V$. Now let $\mathcal{T}_{1} \preceq \mathcal{T}_{2} \preceq \mathcal{T}_{3} \preceq \cdots$ be an ascending sequence of elements of $\mathbb{S}$ and let $d(\mathcal{T})$, the depth of this sequence $\mathcal{T}$, equal the largest depth of all the $G$-stable subgroups which occur as members of the various $\mathcal{T}_{i}$ s. We prove by induction on $d(\mathcal{T})$ that the sequence terminates, the result being trivial if $d(\mathcal{T})=0$ since $V$ is the only $G$-stable subgroup of $V$ having depth $\leq 0$.

Now suppose that $d(\mathcal{T})=n$ and that the result holds for all sequences of smaller depth. Write $\mathcal{T}_{i}=\mathcal{T}_{i}^{\prime} \cup \mathcal{T}_{i}^{\prime \prime}$, where $\mathcal{T}_{i}^{\prime}$ contains the $G$-stable subgroups of depth smaller than $n$ and $\mathcal{T}_{i}^{\prime \prime}$ contains those of depth precisely $n$. Suppose $r \leq s$ and let $A \in \mathcal{T}_{s}^{\prime \prime} \subseteq \mathcal{T}_{s}$. Then $\mathcal{T}_{r} \preceq \mathcal{T}_{s}$ implies that $A$ contains some $B \in \mathcal{T}_{r}$. But $B \subseteq A$ implies that $d(B) \geq d(A)=n$, so the definition of $n=d(\mathcal{T})$ implies that $d(B)=n$ and $A=B$. In other words, if $r \leq s$, then $\mathcal{T}_{r}^{\prime \prime} \supseteq \mathcal{T}_{s}^{\prime \prime}$ and we obtain the decreasing sequence of finite sets $\mathcal{T}_{1}^{\prime \prime} \supseteq \mathcal{T}_{2}^{\prime \prime} \supseteq \mathcal{T}_{3}^{\prime \prime} \supseteq \cdots$ which clearly terminates. By deleting the first few terms if necessary, we can now assume that all $\mathcal{T}_{i}^{\prime \prime}$ are equal.

Again let $r \leq s$ and now take $A \in \mathcal{T}_{s}^{\prime} \subseteq \mathcal{T}_{s}$. Then $\mathcal{T}_{r} \preceq \mathcal{T}_{s}$ implies that $A$ contains some $B \in \mathcal{T}_{r}=\mathcal{T}_{r}^{\prime} \cup \mathcal{T}_{r}^{\prime \prime}$. If $B \in \mathcal{T}_{r}^{\prime \prime}$, then $B \in \mathcal{T}_{s}^{\prime \prime}$ and $A \supseteq B$ violates the irredundancy of the set $\mathcal{T}_{s}$. Thus $B \in \mathcal{T}_{r}^{\prime}$ and we conclude that $\mathcal{T}_{r}^{\prime} \preceq \mathcal{T}_{s}^{\prime}$. Since each $\mathcal{T}_{i}^{\prime}$ is obviously irredundant, we now have a new ascending sequence $\mathcal{T}_{1}^{\prime} \preceq \mathcal{T}_{2}^{\prime} \preceq \mathcal{T}_{3}^{\prime} \preceq \cdots$ in $\mathbb{S}$, and this one has depth smaller than $n$. By induction, this new sequence terminates, and since all $\mathcal{T}_{i}^{\prime \prime}$ are equal, the original sequence $\mathcal{T}$ also terminates.

Finally, we show that the property of being an intersection of augmentation ideals can be lifted from finitely generated submodules to the entire group. 
Lemma 1.9. Let $G$ act on $V$ in such a way that all $G$-sections are infinite, and let $I$ be a $G$-stable ideal of the group algebra $K[V]$. Assume that, for every finitely generated $G$-submodule $W$ of $V$, the $G$-stable ideal $I \cap K[W]$ is a finite intersection of augmentation ideals of $G$-stable subgroups of $W$. Then $I$ is an intersection of augmentation ideals of $G$-stable subgroups of $V$.

Proof. Let $\mathbb{S}$ denote the set of all finitely generated $G$-submodules of $V$. If $X \in \mathbb{S}$, then, by assumption and Lemma $1.4, I \cap K[X]=\bigcap_{i=1}^{n} \omega\left(A_{i} ; X\right)$ is uniquely a finite irredundant intersection of augmentation ideals with each $A_{i}$ a $G$-submodule of $X$. For convenience, write $\mathbb{A}_{X}=\left\{A_{1}, A_{2}, \ldots, A_{n}\right\}$.

For each $X \in \mathbb{S}$ and $A \in \mathbb{A}_{X}$, let $\mathbb{B}_{X, A}$ denote the set of $G$-submodules $B$ of $V$ satisfying:

(i) $B \cap X \subseteq A$, and

(ii) $B \cap Y$ contains a member of $\mathbb{A}_{Y}$ for each $Y \in \mathbb{S}$ with $Y \supseteq X$.

We first prove that each $\mathbb{B}_{X, A}$ is nonempty. To this end, let $\mathbb{S}_{X}$ denote the subset of $\mathbb{S}$ consisting of all $Y$ with $Y \supseteq X$, and consider all "choice" functions $f$ defined on subsets $\mathcal{D}$ of $\mathbb{S}_{X}$ satisfying

$$
f: \mathcal{D} \rightarrow \bigcup_{Y \in \mathbb{S}_{X}} \mathbb{A}_{Y}=\mathbb{T}_{X} \quad \text { and } \quad f(Y) \in \mathbb{A}_{Y}
$$

Let us say that such a function $f$ is "good" if for all finite subsets $\left\{Y_{1}, Y_{2}, \ldots, Y_{m}\right\}$ of $\mathcal{D}$, we have

$$
X \cap\left\langle f\left(Y_{1}\right), f\left(Y_{2}\right), \ldots, f\left(Y_{m}\right)\right\rangle \subseteq A
$$

It is clear that if $f: \mathcal{D} \rightarrow \mathbb{T}_{X}$ is good, then so is the restriction of $f$ to any subset of $\mathcal{D}$. Next, suppose that $Y_{1}, Y_{2}, \ldots, Y_{n} \in \mathbb{S}_{X}$ and let $Z=\left\langle Y_{1}, Y_{2}, \ldots, Y_{n}\right\rangle$. By assumption and Lemma 1.5, there exist some $E \in \mathbb{A}_{Z}$ with $E \cap X=A$. Furthermore, by Lemma 1.5 again, $E \cap Y_{i} \supseteq C_{i}$ for some $C_{i} \in \mathbb{A}_{Y_{i}}$. Thus, if we define

$$
f:\left\{Y_{1}, Y_{2}, \ldots, Y_{m}\right\} \rightarrow \mathbb{T}_{X}
$$

by $f\left(Y_{i}\right)=C_{i}$, then

$$
X \cap\left\langle f\left(Y_{1}\right), f\left(Y_{2}\right), \ldots, f\left(Y_{m}\right)\right\rangle=X \cap\left\langle C_{1}, C_{2}, \ldots, C_{m}\right\rangle \subseteq X \cap E=A
$$

and $f$ is a good function.

Since each $\mathbb{A}_{Y}$ is finite, the Compactness Theorem (see [P, Theorem 6.3.1] for a slightly weaker version of this result) implies that there exists a good function $g: \mathbb{S}_{X} \rightarrow \mathbb{T}_{X}$, and we set $B=\left\langle g(Y) \mid Y \in \mathbb{S}_{X}\right\rangle$. Then $B$ is certainly a $G$-stable subgroup of $V$ and, for all $Y \in \mathbb{S}_{X}$, we have $B \cap Y \supseteq g(Y)$, so $B \cap Y$ contains a member of $\mathbb{A}_{Y}$. In addition, if $v \in X \cap B$, then there exist $Y_{1}, Y_{2}, \ldots, Y_{m} \in \mathbb{S}_{X}$ with $v \in X \cap\left\langle g\left(Y_{1}\right), g\left(Y_{2}\right), \ldots, g\left(Y_{m}\right)\right\rangle \subseteq A$. Thus $X \cap B \subseteq A$, so $B \in \mathbb{B}_{X, A}$ and $\mathbb{B}_{X, A}$ is indeed a nonempty set.

It is now a simple matter to prove that $I$ is equal to $J=\bigcap \omega(B ; V)$, where the latter intersection is over all $X \in \mathbb{S}, A \in \mathbb{A}_{X}$, and $B \in \mathbb{B}_{X, A}$. For this, first note that if $B \in \mathbb{B}_{X, A}$ and if $Y \in \mathbb{S}_{X}$, then $B \cap Y \supseteq C$ for some $C \in \mathbb{A}_{Y}$ by condition (ii). Thus

$$
\omega(B ; V) \cap K[Y]=\omega(B \cap Y ; Y) \supseteq \omega(C ; Y) \supseteq I \cap K[Y]
$$

and, since this holds for all such $Y$, it follows that $\omega(B ; V) \supseteq I$. Consequently, $J=\bigcap \omega(B ; V) \supseteq I$. Conversely, let $X \in \mathbb{S}$ and let $\mathbb{A}_{X}=\left\{A_{1}, A_{2}, \ldots, A_{n}\right\}$. Then, 
for each $i, \mathbb{B}_{X, A_{i}} \neq \emptyset$, so we can choose some $B_{i}$ in this set, and condition (i) yields

$$
\begin{aligned}
K[X] \cap J & \subseteq K[X] \cap \bigcap_{i=1}^{n} \omega\left(B_{i} ; V\right) \\
& =\bigcap_{i=1}^{n} \omega\left(B_{i} \cap X ; X\right) \subseteq \bigcap_{i=1}^{n} \omega\left(A_{i} ; X\right)=K[X] \cap I .
\end{aligned}
$$

Since this holds for all $X \in \mathbb{S}$, we have the reverse inclusion, and therefore $I=J$ is a suitable intersection.

\section{LOCALLY FINITE FIELDS}

We continue to assume that $K$ is an arbitrary field. In addition, we take $F$ to be a locally-finite field and we let $V$ be an $F$-vector space, viewed multiplicatively. Then $V$ is an elementary abelian $p$-group, where $p=\operatorname{char} F$, and $G=F^{\bullet}$ acts on $V$ in a natural manner. The following extension of [BE Lemma 9] handles the case where both $F$ and $V$ are finite.

Lemma 2.1. Let $F$ be a finite field and let $V$ be a finite-dimensional $F$-vector space, viewed multiplicatively. Assume that $\operatorname{char} F \neq \operatorname{char} K$. Then $G=F^{\bullet}$ acts on $V$ and every $G$-stable ideal of $K[V]$ contained in $\omega(V ; V)$ is a finite intersection of augmentation ideals $\omega(A ; V)$ with $A$ a $G$-stable subgroup of $V$.

Proof. Suppose first that $K$ is algebraically closed or at least that it contains a primitive $p$ th root of unity for $p=\operatorname{char} F$. Let $\Lambda=\Lambda(V)$ be the set of nonprincipal irreducible representations of $K[V]$ and let $\left\{e_{\lambda} \mid \lambda \in \Lambda\right\}$ be the corresponding set of primitive idempotents in $K[V]$. Then we know that the latter are orthogonal idempotents and that $\omega(V ; V)=\oplus \sum_{\lambda \in \Lambda} K e_{\lambda}$. Furthermore, if $I$ is an ideal of $K[V]$ contained in $\omega(V ; V)$, then $I=\oplus \sum_{\lambda \in \Lambda} I e_{\lambda}$ and, for each $\lambda, I e_{\lambda}$ is either 0 or $K e_{\lambda}$. Thus $I$ is uniquely determined by its support, namely $\operatorname{supp} I=\left\{\lambda \in \Lambda \mid e_{\lambda} \in I\right\}$. Indeed, $I=\oplus \sum_{\lambda \in \operatorname{supp} I} K e_{\lambda}$, and $I$ is $G$-stable if and only if supp $I$ is $G$-stable.

Let $\mathcal{O}_{1}, \mathcal{O}_{2}, \ldots, \mathcal{O}_{m}$ be the orbits of the natural action of $G$ on $\Lambda$, and let $I_{i}$ be the ideal of $K[V]$ contained in $\omega(V ; V)$ and satisfying $\operatorname{supp} I_{i}=\Lambda \backslash \mathcal{O}_{i}$. Then, the $I_{i}$ 's are clearly the maximal $G$-stable ideals of $K[V]$ properly contained in $\omega(V ; V)$. Furthermore, if $I$ is a $G$-stable ideal of $K[V]$ with $I \subseteq \omega(V ; V)$ and $\operatorname{supp} I=\Lambda \backslash \bigcup_{i \in \mathcal{I}} \mathcal{O}_{i}$, then clearly $I=\bigcap_{i \in \mathcal{I}} I_{i}$. Thus, it suffices to show that each $I_{i}$ is a suitable augmentation ideal.

To this end, let $|F|=q$ and $|V|=q^{n}$. Since the split extension $V \rtimes G$ is a Frobenius group, it follows that $G$ acts in a semiregular fashion, that is with full orbit sizes, on $\Lambda$. Thus, since $|\Lambda|=q^{n}-1$, we see that the number of orbits $\mathcal{O}_{i}$ is precisely equal to $m=\left(q^{n}-1\right) /(q-1)$ and this is the same as the number of $F$-subspaces of $V$ of codimension 1 . In particular, if $n=1$, then $m=1$ and we see that $\omega(V ; V)$ is the unique nonzero $G$-stable ideal of $K[V]$ contained in $\omega(V ; V)$. For the general case, let $A_{1}, A_{2}, \ldots, A_{m}$ be the $m$ subspaces of $V$ of codimension 1. Then each $\omega\left(A_{i} ; V\right)$ is $G$-stable and

$$
\omega(V ; V) / \omega\left(A_{i} ; V\right)=\omega\left(V / A_{i} ; V / A_{i}\right) \triangleleft K\left[V / A_{i}\right] .
$$

But $\operatorname{dim}_{F} V / A_{i}=1$, so the above $n=1$ observation implies that $\omega\left(V / A_{i} ; V / A_{i}\right)$ is a minimal nonzero $G$-stable ideal of $K\left[V / A_{i}\right]$. Consequently, each $\omega\left(A_{i} ; V\right)$ is a $G$-stable ideal of $K[V]$ maximal subject to being properly contained in $\omega(V ; V)$. In other words, we have constructed $m$ distinct $G$-stable ideals $\omega\left(A_{i} ; V\right)$, all maximal 
subject to being properly contained in $\omega(V ; V)$. Since this obviously accounts for all the ideals $I_{1}, I_{2}, \ldots, I_{m}$, the algebraically closed case is proved.

Finally, let $K$ be arbitrary, subject to $\operatorname{char} K \neq \operatorname{char} F$, and let $K^{\prime}$ be its algebraic closure. Note that if $J$ is any ideal of $K[V]$, then $J^{\prime}=J \cdot K^{\prime}=J \cdot K^{\prime}[V]$ is an ideal of $K^{\prime}[V]$. Furthermore, since $K$ is a $K$-module direct summand of $K^{\prime}$, it follows that $J=J^{\prime} \cap K[V]$. In particular, since $\omega_{K}(A ; V)^{\prime}=\omega_{K^{\prime}}(A ; V)$, we see that $\omega_{K^{\prime}}(A ; V) \cap K[V]=\omega_{K}(A ; V)$. Now $I$ is a $G$-stable ideal of $K[V]$ contained in $\omega_{K}(V ; V)$, so $I^{\prime}$ is a $G$-stable ideal of $K^{\prime}[V]$ contained in $\omega_{K^{\prime}}(V ; V)$. Thus, by the algebraically closed result, $I^{\prime}=\bigcap_{1}^{t} \omega_{K^{\prime}}\left(B_{i} ; V\right)$ for suitable $G$-stable subgroups $B_{i}$ of $V$, and consequently

$$
I=I^{\prime} \cap K[V]=\bigcap_{1}^{t}\left(\omega_{K^{\prime}}\left(B_{i} ; V\right) \cap K[V]\right)=\bigcap_{1}^{t} \omega_{K}\left(B_{i} ; V\right),
$$

as required.

It is now a simple matter, using the results of the preceding section, to lift this lemma to the infinite situation. Note that, if $V$ is an $F$-vector space and if $G=F^{\bullet}$, then the $G$-stable subgroups of $V$ are precisely the $F$-subspaces of $V$. In particular, when $F$ is infinite, each $G$-section of $V$ is a nontrivial $F$-vector space and hence is also infinite.

Lemma 2.2. Let $F$ be an infinite-locally finite field and suppose that $V$ is an $F$ vector space, viewed multiplicatively. Assume that $\operatorname{char} K \neq \operatorname{char} F$. Then $G=F^{\bullet}$ acts on $V$ and every $G$-stable ideal of $K[V]$ is an intersection of augmentation ideals $\omega(A ; V)$ with $A$ a $G$-stable subgroup of $V$.

Proof. Assume first that $V=F^{n}$ is finite dimensional, and let $F_{1} \subseteq F_{2} \subseteq \cdots$ be an ascending union of finite subfields of $F$ with $\bigcup_{1}^{\infty} F_{i}=F$. If $G_{i}=F_{i}^{\bullet}$ and $V_{i}=F_{i}^{n}$, then it is easy to see that $\left\{\left(V_{i}, G_{i}\right) \mid i=1,2, \ldots\right\}$ is a local system for $(V, G)$. Now if $I$ is any $G$-stable ideal of $K[V]$ contained in $\omega(V ; V)$, then Lemma 1.1(i) implies that, for each $i, K\left[V_{i}\right] \cap I$ is a $G_{i}$-stable ideal of $K\left[V_{i}\right]$ contained in $\omega\left(V_{i} ; V_{i}\right)$. Hence, by Lemma 2.1, each $K\left[V_{i}\right] \cap I$ is a finite intersection of augmentation ideals $\omega\left(A ; V_{i}\right)$ with $A$ a $G_{i}$-stable subgroup of $V_{i}$, and it follows from Lemma 1.2 that $I$ is an intersection of augmentation ideals of $G$-stable subgroups of $V$.

Next, let $I$ be an arbitrary $G$-stable ideal of $K[V]$. If $I \supseteq \omega(V ; V)$, then either $I=\omega(V ; V)$ or $I=K[V]$, and the latter is an empty intersection of augmentation ideals. On the other hand, if $I \nsupseteq \omega(V ; V)$, then $J=I \cap \omega(V ; V)$ is a $G$-stable ideal of $K[V]$ properly contained in $\omega(V ; V)$. By the above, $J$ is contained in some augmentation ideal $\omega(B ; V)$ with $B$ a $G$-stable subgroup of $V$ properly smaller than $V$. In particular, $\omega(B ; V) \nsupseteq \omega(V ; V)$. But $\omega(B ; V) \supseteq J=I \cap \omega(V ; V)$ and $\omega(B ; V)$ is a $G$-prime ideal by Lemma $1.3($ ii). Hence $I \subseteq \omega(B ; V) \subseteq \omega(V ; V)$ and the result of the preceding paragraph applies to $I$.

Finally, if $V$ is an arbitrary $F$-vector space, then the finitely generated $G$ submodules of $V$ are precisely the finite-dimensional $F$-subspaces of $V$. With this, the preceding remarks and Lemma 1.9 yield the result.

We can now offer the

Proof of Theorem A. If $V$ is a finite-dimensional $F$-vector space, then it is clear that $V$ has $G$-composition length equal to $\operatorname{dim}_{F} V<\infty$. Now, by Lemma 2.2, every $G$-stable ideal of $K[V]$ is an intersection of augmentation ideals $\omega(A ; V)$ with 
$A$ a $G$-stable subgroup of $V$. Next, Lemma 1.6 implies that every such ideal is a finite intersection of suitable augmentation ideals. We can then assume that these intersections are irredundant, and conclude from Lemma 1.4 that the corresponding $G$-stable subgroups are unique. Finally, by Lemma 1.8, the set of all such ideals satisfies the ascending chain condition.

Let $\mathfrak{G}$ be a group acting on a set $\Lambda$. Then we recall that an element $\lambda \in \Lambda$ is said to be $\mathfrak{G}$-orbital if it has finitely many $\mathfrak{G}$-conjugates or equivalently if the stabilizer in $\mathfrak{G}$ of $\lambda$ has finite index in the group.

Lemma 2.3. Let $F$ be an infinite locally-finite field, let $V$ be a finite-dimensional $F$-vector space, viewed multiplicatively, and let $\mathfrak{G}$ be a group which acts on $V$ and contains $F^{\bullet}$, in its natural action, as a normal subgroup. If char $K \neq \operatorname{char} F$, then every $\mathfrak{G}$-stable ideal of $K[V]$ is uniquely an irredundant intersection $\bigcap_{A \in \mathfrak{A}} \omega(A ; V)$, where $\mathfrak{A}$ is a finite $\mathfrak{G}$-stable set of $F$-subspaces of $V$. In particular, each $A \in \mathfrak{A}$ is an $F^{\bullet}$-stable, $\mathfrak{G}$-orbital subgroup of $V$.

Proof. If $I$ is $\mathfrak{G}$-stable, then it is $F^{\bullet}$-stable. Hence by Theorem A, $I$ can be written uniquely as the irredundant intersection $I=\bigcap_{i=1}^{m} \omega\left(A_{i} ; V\right)$ where each $A_{i}$ is $F^{\bullet}$ stable. In particular, if $g \in \mathfrak{G}$, then since $I$ is $\mathfrak{G}$-stable, we have

$$
\bigcap_{i=1}^{m} \omega\left(A_{i} ; V\right)=I=I^{g}=\bigcap_{i=1}^{m} \omega\left(A_{i}^{g} ; V\right) .
$$

But $F^{\bullet}$ is normal in $\mathfrak{G}$, so each $A_{i}^{g}$ is certainly an $F^{\bullet}$-stable subgroup of $V$. Hence, by uniqueness, $\left\{A_{1}^{g}, A_{2}^{g}, \ldots, A_{m}^{g}\right\}=\left\{A_{1}, A_{2}, \ldots, A_{m}\right\}$ and consequently $\mathfrak{G}$ permutes the finite set $\mathfrak{A}=\left\{A_{1}, A_{2}, \ldots, A_{m}\right\}$.

With this, we can consider a question posed in [HZ]. Namely, let $F$ be a locallyfinite field and suppose that $F$ is generated by two infinite subfields $F_{1}$ and $F_{2}$. If $V$ is an $F$-vector space and if $I$ is an ideal of $K[V]$ stable under both $F_{1}^{\bullet}$ and $F_{2}^{\bullet}$, must $I$ also be stable under $F^{\bullet}$ ? We obtain an affirmative answer, at least when $F_{1} \cap F_{2}$ is infinite.

Lemma 2.4. Let $F$ be a locally finite field generated by the two subfields $F_{1}$ and $F_{2}$ with $F_{1} \cap F_{2}$ infinite. Let $V$ be an $F$-vector space, viewed multiplicatively, and let $\operatorname{char} K \neq \operatorname{char} F$. If $I$ is an ideal of $K[V]$ stable under both $F_{1}^{\bullet}$ and $F_{2}^{\bullet}$, then $I$ is stable under $F^{\bullet}$.

Proof. Let $I$ be as above and choose $\alpha \in I$ and $f \in F^{\bullet}$. The goal is to show that $\alpha^{f} \in I$. Now $f \in\left\langle F_{1}, F_{2}\right\rangle$, so there exists a subfield $\tilde{F}_{2}$ of $F_{2}$ with $f \in\left\langle F_{1}, \tilde{F}_{2}\right\rangle=\tilde{F}$ and with degree $\left(\tilde{F}_{2}: F_{1} \cap F_{2}\right)<\infty$. Furthermore, there exists a finite-dimensional $\tilde{F}$-subspace $\tilde{V}$ of $V$ with $\alpha \in I \cap K[\tilde{V}]=\tilde{I}$. Since $\left(\tilde{F}: F_{1}\right) \leq\left(\tilde{F}_{2}: F_{1} \cap F_{2}\right)<\infty$ and $\operatorname{dim}_{\tilde{F}} \tilde{V}<\infty$, we see that $\tilde{V}$ is also finite dimensional as an $F_{1}$-space. Now $\tilde{I}$ is certainly both $F_{1}^{\bullet}$ - and $\tilde{F}_{2}^{\bullet}$-stable and $F_{1}$ is an infinite field, so Lemma 2.3, applied to the group $F_{1}^{\bullet} \tilde{F}_{2}^{\bullet}$, implies that $\tilde{I}=\bigcap_{A \in \mathfrak{A}} \omega(A ; \tilde{V})$ where each $A \in \mathfrak{A}$ is an $F_{1}^{\bullet}$-stable, $\tilde{F}_{2}^{\bullet}$-orbital subgroup of $\tilde{V}$. By assumption, $F_{1} \cap F_{2}$ is infinite, so $\tilde{F}_{2}$ is infinite, and therefore any subgroup of finite index in $\tilde{F}_{2}^{\bullet}$ must additively generate the field $\tilde{F}_{2}$. In particular, since each $A \in \mathfrak{A}$ is $\tilde{F}_{2}^{\bullet}$-orbital, it follows that each such $A$ is $\tilde{F}_{2}^{\bullet}$-stable. Furthermore, since each $A$ is $F_{1}^{\bullet}$-stable and since $\tilde{F}=\left\langle F_{1}, \tilde{F}_{2}\right\rangle$, we see that each $A \in \mathfrak{A}$ is $\tilde{F}^{\bullet}$-stable. Thus, $\tilde{I}$ is an intersection of the $\tilde{F}^{\bullet}$-stable 
augmentation ideals $\omega(A ; \tilde{V})$, so $\tilde{I}$ is also $\tilde{F}^{\bullet}$-stable. Since $\alpha \in \tilde{I}$ and $f \in \tilde{F}^{\bullet}$, we conclude that $\alpha^{f} \in \tilde{I} \subseteq I$, as required.

It follows from the above and a trivial induction that if $F=\left\langle F_{1}, F_{2}, \ldots\right\rangle$ is locally finite and if $F_{n+1} \cap\left\langle F_{1}, F_{2}, \ldots, F_{n}\right\rangle$ is infinite for all $n \geq 1$, then an ideal of $K[V]$ is $F^{\bullet}$-stable if and only if it is $F_{n}^{\bullet}$-stable for all $n$.

Finally, as we observed in the introduction, Theorem A is essentially the locallyfinite analog of [BE, Proposition 6], a result on the field of rational numbers. Both of these facts will be used in Part II of this work to handle arbitrary division rings.

\section{REFERENCES}

[BE] C. J. B. Brookes and D. M. Evans, Augmentation modules for affine groups, Math. Proc. Cambridge Philos. Soc. 130 (2001), 287-294. CMP 2001:06

[HZ] B. Hartley and A. E. Zalesskiı̌, Group rings of periodic linear groups, unpublished note (1995).

[P] D. S. Passman, The Algebraic Structure of Group Rings, Wiley-Interscience, New York, 1977. MR 81d:16001

[PZ1] D. S. Passman and A. E. Zalesskiॅ, Invariant ideals of abelian group algebras and representations of groups of Lie type, Trans. AMS 353 (2001), 2971-2982.

[Z] A. E. Zalesski1̌, Group rings of simple locally finite groups, Finite and Locally Finite Groups, Kluwer, Dordrecht, 1995, pp. 219-246. MR 96k:16044

Department of Mathematics, University of Wisconsin, Madison, Wisconsin 53706

E-mail address: Passman@math.wisc.edu

School of Mathematics, University of East Anglia, Norwich NR4 7TJ, United KingDOM

E-mail address: A.Zalesskii@uea.ac.uk 